ICD-10 diagnoses and medications) was assessed during the 12-months following AAP initiation. Cohorts with and without EPS were defined. Demographics, clinical characteristics, and healthcare resource use and costs over 12 months following the first EPS claim (EPS) or randomly assigned index date (Non-EPS) were assessed.

Results. A total of 11,642 patients with schizophrenia were identified; $21.2 \%$ developed EPS in the 12-months following AAP initiation. EPS and Non-EPS cohorts included 2,295 (mean age $38,61 \%$ male, CCI 0.6 ) and 5,607 (mean age $39,57 \%$ male, CCI 0.7) patients, respectively. Over the 12 -month post-index period, EPS cohort had significantly higher rates of all-cause (30.2\% vs. $24.6 \%, \mathrm{p}<0.001)$ and schizophrenia-related hospitalizations $(22.5 \%$ vs. $12.9 \%, \mathrm{p}<0.001)$ and schizophrenia-related emergency room visits $(25.5 \%$ vs. $16.7 \%, \mathrm{p}<0.001)$ compared to Non-EPS cohort. All-cause $(\$ 25,911$ vs. $\$ 21,550, \mathrm{p}<0.001)$ and schizophrenia-related healthcare costs $(\$ 12,134$ vs. $\$ 6,230$, $\mathrm{p}<0.001)$ were significantly higher in EPS vs. Non-EPS cohort. Conclusions. In the 12 months following AAP initiation, over $20 \%$ of schizophrenia patients developed EPS, which was associated with increased healthcare resource utilization and costs. Treatment options that minimize EPS may reduce the economic burden of schizophrenia.

Funding. Sunovion Pharmaceuticals Inc.

\section{Is this Withdrawal or Intoxication? Case Report Regarding Complications of Unregulated Use of Tianeptine, Etizolam, and Phenibut in the USA}

\section{Sadia B. Ghani, MD, Eric Taylor, MD and \\ Siddesh Gopalakrishnan, MD}

University of Arizona, Department of Psychiatry, Tucson, AZ, USA

Presenting Author: Sadia B. Ghani, Eric Taylor, Siddesh Gopalakrishnan

\section{Abstract}

Background. The internet allows easy access for the sales of psychoactive agents that are not regulated by the FDA. Some of those agents are used to help manage anxiety, depression and sleep, such as tianeptine, etizolam, and phenibut. These medications have the potential for abuse and potentially leading to altered mental status when intoxicated or withdrawing. This presents a challenge to clinicians who may not be aware of availability of such substances. Available literature has discussed the use of above substances individually, but how do you treat if there is use of more than one substance with different mechanisms of actions? Here we present a case of an adult male who has used all three agents simultaneously, leading to a hospital admission.
\end{abstract}

Case History. A 32-year-old male presented to the emergency department (ED) for altered mental status (AMS). He has a documented history of anxiety but was never treated with prescription medications. No history of substance use was documented. He was self-medicating with concurrent use of tianeptine (atypical antidepressant with mu agonist properties,) phenibut (GABA mimetic) and etizolam (a benzodiazepine-like agent). During his stay, he was agitated and delirious with reports of visual hallucinations. Neuroimaging and lab studies were within normal limits, EEG showed no seizure activity. Over the course of his hospital stay, he was started on Depakote for agitation, a Valium taper for suspected benzodiazepine withdrawal and prevention of seizures, Seroquel for delirium, and baclofen for suspected GABAergic withdrawal symptoms. The patient's AMS improved and he was discharged on hospital day 10.

Conclusions. This case illustrates the difficulty managing poly-substance use/abuse and stresses the importance for physicians to screen for psychoactive agents purchased over the internet or over the counter to improve treatment outcomes. Continued discussions with patients regarding risks/ benefits of use of such substances would be beneficial and help increase awareness.

\section{Safety of Using a Combinatorial Pharmacogenomic Test for Patients with Major Depressive Disorder in the GUIDED trial}

\author{
Sagar V. Parikh, MD' ${ }^{1}$, Gabriela K. Khazanov, $\mathrm{PhD}^{2}$, \\ Michael E. Thase, $M^{2}$, Anthony J. Rothschild, $M^{3}{ }^{3}$, \\ Boadie W. Dunlop, $\mathrm{MD}^{4}$, Charles DeBattista, $\mathrm{DMH}, \mathrm{MD}^{5}$, \\ Charles R. Conway, $\mathrm{MD}^{6}$, Brent P. Forester, $\mathrm{MD}, \mathrm{MSc}^{7}$, \\ Richard C. Shelton, $\mathrm{MD}^{8}$, Matthew Macaluso, $\mathrm{DO}^{9}$, \\ James Li, PhD ${ }^{10}$, Kunbo Yu, MS ${ }^{10}$, \\ Michael R. Jablonski, $\mathrm{PhD}^{10}$, Stephanie Meek, $\mathrm{PhD}^{11}$ and \\ John F. Greden, MD ${ }^{1}$
}

\footnotetext{
${ }^{1}$ University of Michigan, Comprehensive Depression Center and Department of Psychiatry, and National Network of Depression Centers, Ann Arbor, MI, USA, ${ }^{2}$ University of Pennsylvania, Perelman School of Medicine, and the Corporal Michael Crescenz VAMC, Philadelphia, PA, USA, ${ }^{3}$ University of Massachusetts Medical School and UMass Memorial Healthcare, Worcester, MA, USA, ${ }^{4}$ Emory University School of Medicine, Department of Psychiatry and Behavioral Sciences, Atlanta, GA, USA, ${ }^{5}$ Stanford University School of Medicine, Stanford, CA, USA, ${ }^{6}$ Washington University School of Medicine, St. Louis, MO, USA, ${ }^{7}$ McLean Hospital, Belmont, MA, USA, ${ }^{8}$ The University of Alabama at Birmingham, Birmingham, AL, USA, ${ }^{9}$ University of Kansas School of Medicine-Wichita, Wichita, KS, USA, ${ }^{10}$ Myriad Neuroscience, Mason, OH, USA, and ${ }^{11}$ Myriad Genetics, Inc., Salt Lake City, UT, USA
}

Presenting Author: Sagar V. Parikh 